\title{
Generalized orthonormal moment tensor decomposition and its source-type diagram
}

\author{
Ting-Chung Huang (D) - Yih-Min Wu
}

Received: 10 December 2019 / Accepted: 10 August 2020 / Published online: 16 September 2020

(C) The Author(s) 2020

\begin{abstract}
Moment tensor decomposition is a method for deriving the isotropic (ISO), double-couple (DC), and compensated linear vector dipole (CLVD) components from a seismic moment tensor. Currently, there are two families of methods, namely, standard moment tensor decomposition and Euclidean moment tensor decomposition. Although both methods can usually provide workable solutions, there are some minor inconsistencies between the two methods: an equality inconsistency that occurs in standard moment tensor decomposition and the pure CLVD unity and flip basis inconsistency encountered in Euclidean moment tensor decomposition. Moreover, there is a sign problem
\end{abstract}

T.-C. Huang $(\bowtie) \cdot$ Y.-M. Wu

Department of Geosciences, National Taiwan University, 1, Sec. 4, Roosevelt Road, 106, Taipei, Taiwan

e-mail: tingchunghuang@gmail.com

Y.-M. Wu

e-mail: drymwu@ntu.edu.tw

Y.-M. Wu

NTU Research Center for Future Earth, National Taiwan

University, Taipei, 10617, Taiwan

Y.-M. Wu

Institute of Earth Sciences, Academia Sinica 128, Sec. 2,

Academia Road, Nangang, Taipei, 11529, Taiwan

Y.-M. Wu

National Center for Research on Earthquake Engineering, National Applied Research Laboratories, No. 200, Section

3, Xinhai Rd, Taipei, 106, Taiwan when disentangling the CLVD component from a DCdominated case. To address these minor inconsistencies, we propose a new moment tensor decomposition method inspired by both previous methods. The new method can not only avoid all these minor inconsistencies but also withstand deviations in ISO- or CLVD-dominated cases when using source-type diagrams.

Keywords Focal mechanism · Compensated linear vector dipole $\cdot$ Theoretical seismology

\section{Article highlights}

- We examine the inconsistencies among current moment tensor decomposition methods.

- Based on insights from observations of these inconsistencies, we propose an overall consistent moment tensor decomposition method.

- We discuss some interesting features of the proposed method and how to extend it to additional applications.

\section{Introduction}

The seismic moment tensor, which describes the basic characteristics of an earthquake, constitutes one of the most crucial quantities derived from point-source approximations. The spectral decomposition theorem guarantees that the moment tensor must contain real eigenvalues. To associate a physical process with an earthquake, the three-eigenvalue tuple 
is decomposed into three elementary components: the isotropic (ISO) component, the double-couple (DC) component, and the compensated linear vector dipole (CLVD) (Knopoff and Randall 1970) component. However, there is no universal method for decomposition. In recent years, some interest in revisiting the decomposition methods has arisen due to progress in observing non-DC microseismic events (Miller et al. 1998; Eaton and Forouhideh 2010; Chapman 2019).

Moment tensors can be decomposed using a variety of methods that, roughly speaking, can be divided into two families: standard methods and Euclidean methods. The standard method family (Hudson et al. 1989; Jost and Herrmann 1989; Julian et al. 1998; Vavryčuk 2015) utilizes the difference between the basis and its inner product vector to generate the correct magnitude of a pure CLVD moment tensor. There have been many recent developments and new variations. For example, there is an effort to pursue a uniformly distributed parametrization (Chapman 2019). In contrast, the Euclidean method family (Chapman and Leaney 2012; Zhu and Ben-Zion 2013) maintains the orthogonality of the three bases, and thus, the calculations can be performed through a series of projections along the three bases, thereby generating the exact expansion of a moment tensor. A series of papers discusses the 3D geometry of its unique lune-shaped parameter space (Tape and Tape 2012a; 2015; 2019). Finally, there are some efforts to reparametrize the decomposition results, which can also result in some inconsistencies. There is a review paper that summarizes all of the currently proposed parametrization schemes for moment tensor decomposition (Aso et al. 2016).

\subsection{Standard moment tensor decomposition}

Here, we summarize the standard moment tensor decomposition method. The family of standard moment tensor decomposition is large and widely used. Roughly speaking, any decomposition that has a quadrilateral scale plot likely belongs to this family. We choose to use one specific setup adapted from Vavryčuk (2015) to represent the entire family. All of the quantitative phenomena discussed below also apply to other methods within the standard family. The choice of this specific setup is done without loss of generality.
The first step is to diagonalize the moment tensor into three eigenvalues, which are then ordered into $M_{1}^{*} \geq M_{2}^{*} \geq M_{3}^{*}$. Because all of the off-diagonal terms are zero, we can effectively group the ordered eigenvalues into a vector,

$\mathbf{M}^{*}=\left[\begin{array}{c}M_{1}^{*} \\ M_{2}^{*} \\ M_{3}^{*}\end{array}\right]$,

and then we can transform the moment tensor decomposition process into the decomposition of vector $\mathbf{M}^{*}$.

\subsubsection{The type I case}

When

$M_{1}^{*}+M_{3}^{*}-2 M_{2}^{*} \geq 0$,

the basis vectors are

$E_{I S O}^{S}=\left[\begin{array}{l}1 \\ 1 \\ 1\end{array}\right], E_{D C}^{S}=\left[\begin{array}{l}1 \\ 0 \\ -1\end{array}\right], E_{C L V D}^{S}=\frac{1}{2}\left[\begin{array}{l}2 \\ -1 \\ -1\end{array}\right]$,

and the three decomposition components are

$$
\begin{aligned}
M_{I S O}^{S} & =\frac{1}{3}\left(M_{1}^{*}+M_{2}^{*}+M_{3}^{*}\right)=\frac{1}{3} \mathbf{M}^{*} \cdot E_{I S O}^{S}, \\
M_{C L V D}^{S} & =\frac{2}{3}\left(M_{1}^{*}+M_{3}^{*}-2 M_{2}^{*}\right) \neq \frac{2}{3} \mathbf{M}^{*} \cdot E_{C L V D}^{S}, \\
M_{D C}^{S} & =M_{2}^{*}-M_{3}^{*} \neq \mathbf{M}^{*} \cdot E_{D C}^{S} .
\end{aligned}
$$

Note that except for the ISO component, all other components are not proportional to the projection along the basis vectors. Additionally, note how the components of the basis vectors are kept preordered.

\subsubsection{The type II case}

When

$M_{1}^{*}+M_{3}^{*}-2 M_{2}^{*}<0$,

the basis vectors are

$E_{I S O}^{S}=\left[\begin{array}{l}1 \\ 1 \\ 1\end{array}\right], E_{D C}^{S}=\left[\begin{array}{l}1 \\ 0 \\ -1\end{array}\right], E_{C L V D}^{S}=\frac{1}{2}\left[\begin{array}{l}1 \\ 1 \\ -2\end{array}\right]$, 
and the three decomposition components are

$$
\begin{aligned}
M_{I S O}^{S} & =\frac{1}{3}\left(M_{1}^{*}+M_{2}^{*}+M_{3}^{*}\right)=\frac{1}{3} \mathbf{M}^{*} \cdot E_{I S O}^{S}, \\
M_{C L V D}^{S} & =\frac{2}{3}\left(M_{1}^{*}+M_{3}^{*}-2 M_{2}^{*}\right) \neq \frac{2}{3} \mathbf{M}^{*} \cdot E_{C L V D}^{S}, \\
M_{D C}^{S} & =M_{1}^{*}-M_{2}^{*} \neq \mathbf{M}^{*} \cdot E_{D C}^{S} .
\end{aligned}
$$

\subsubsection{Scale factors}

The scalar moment is defined as

$M_{0}^{S}=\left|M_{I S O}^{S}\right|+\left|M_{C L V D}^{S}\right|+M_{D C}^{S}$,

where the unity is preserved in the most straightforward way. However, there are different definitions defining the scalar moment. For example, Vavryčuk (2005) used two different spectral norm moments $M$, $\bar{M}$, to maintain the unity equation.

In addition, the three scale factors are defined as

$$
C_{I S O}^{S}=\frac{M_{I S O}^{S}}{M_{0}^{S}}, C_{D C}^{S}=\frac{M_{D C}^{S}}{M_{0}^{S}}, C_{C L V D}^{S}=\frac{M_{C L V D}^{S}}{M_{0}^{S}} .
$$

Moreover, the unity equation of the three scale factors is linear,

$$
\left|C_{I S O}^{S}\right|+C_{D C}^{S}+\left|C_{C L V D}^{S}\right|=1
$$

\subsection{The equality inconsistency in standard moment tensor decomposition}

The equality inconsistency in standard moment tensor decomposition occurs in either the type II or type I case (type II in our setup), and it can be demonstrated with ease by considering a pure CLVD. Considering an ordered eigenvalue vector of the moment tensor $\mathbf{M}^{*}=(1 / 2,1 / 2,-1)^{T}$, which is essentially the eigenvalue vector used in Riedesel and Jordan (1989), the only nonzero coefficient is $M_{\mathrm{CLVD}}^{S}=$ -1 . Although the sign and magnitude of this coefficient seem reasonable, the expression for the full decomposition reveals the abovementioned issue,

$$
\left[\begin{array}{c}
1 / 2 \\
1 / 2 \\
-1
\end{array}\right] \neq-1\left[\begin{array}{c}
1 / 2 \\
1 / 2 \\
-1
\end{array}\right]=M_{\mathrm{CLVD}}^{S} E_{\mathrm{CLVD}}^{S}
$$

Note that for the method in Hudson et al. (1989), the equality inconsistency is observed in the type II case, for example, $\mathbf{M}^{*}=(2,-1,-1)^{T}$. The standard method family can only fix one case at a time and will inevitably produce an equality inconsistency in the other case. It cannot have both cases fixed at the same time. Moreover, one may think that the equality issue can be easily solved by switching the sign of the type II CLVD basis vector. This will work; however, it will introduce new inconsistencies. Specifically, changing the sign of the type II CLVD will break the preordering and assign the type II CLVD to the type I case. It is common in both families that a quick fix will simply introduce new inconsistencies of some kind. Mathematically speaking, this equality inconsistency is deeply associated with the fact that (1) the standard methods use nonorthogonal bases and (2) two out of three components are not the projection along its bases.

\subsection{Euclidean moment tensor decomposition}

Here, we summarize the Euclidean moment tensor decomposition method. Roughly speaking, any decomposition that has a lune shape scale plot with the ISO direction longer than the CLVD direction likely belongs to this family. We choose to use one plain setup adapted from Vavryčuk (2015) to represent the entire family. Again, the choice of this specific setup is done without loss of generality.

Starting with preordered eigenvalues $M_{1}^{*}, M_{2}^{*}$, and $M_{3}^{*}$, the basis vectors are

$$
E_{I S O}^{E}=\sqrt{\frac{2}{3}}\left[\begin{array}{l}
1 \\
1 \\
1
\end{array}\right], E_{D C}^{E}=\left[\begin{array}{l}
1 \\
0 \\
-1
\end{array}\right], E_{C L V D}^{E}=\frac{1}{\sqrt{3}}\left[\begin{array}{l}
1 \\
-2 \\
1
\end{array}\right],
$$

and the three decomposition components can be expressed as a series of projections along the basis vectors,

$$
\begin{aligned}
M_{I S O}^{E} & =\frac{1}{\sqrt{6}}\left(M_{1}^{*}+M_{2}^{*}+M_{3}^{*}\right)=\frac{1}{2} \mathbf{M}^{*} \cdot E_{I S O}^{E} \\
M_{C L V D}^{E} & =\frac{1}{2 \sqrt{3}}\left(M_{1}^{*}+M_{3}^{*}-2 M_{2}^{*}\right)=\frac{1}{2} \mathbf{M}^{*} \cdot E_{C L V D}^{E}, \\
M_{D C}^{E} & =\frac{1}{2}\left(M_{1}^{*}-M_{3}^{*}\right)=\frac{1}{2} \mathbf{M}^{*} \cdot E_{D C}^{E}
\end{aligned}
$$

Note that there is a sign flip in the CLVD basis vector. 
The scalar moment is defined as

$$
\begin{aligned}
M_{0}^{E} & =\sqrt{\frac{\left(M_{1}^{*}\right)^{2}+\left(M_{2}^{*}\right)^{2}+\left(M_{3}^{*}\right)^{2}}{2}} \\
& =\sqrt{\left(M_{I S O}^{E}\right)^{2}+\left(M_{C L V D}^{E}\right)^{2}+\left(M_{D C}^{E}\right)^{2}},
\end{aligned}
$$

where the Euclidean definition of the scalar moment follows the notation of a previous study (Silver and Jordan 1982), and the extra $1 / 2$ follows the notation from Aki and Richards (2002) to normalize pure DC to 1 . In addition, the three scale factors are defined as

$$
C_{I S O}^{E}=\frac{M_{I S O}^{E}}{M_{0}^{E}}, C_{D C}^{E}=\frac{M_{D C}^{E}}{M_{0}^{E}}, C_{C L V D}^{E}=\frac{M_{C L V D}^{E}}{M_{0}^{E}} .
$$

Moreover, the unity equation of the three scale factors becomes quadratic,

$\left(C_{I S O}^{E}\right)^{2}+\left(C_{D C}^{E}\right)^{2}+\left(C_{C L V D}^{E}\right)^{2}=1$

1.4 The pure CLVD unity and flip basis inconsistency in Euclidean moment tensor decomposition

The pure CLVD unity and basis choice inconsistency in Euclidean moment tensor decomposition occurs in the pure CLVD case. Applying Euclidean decomposition to a pure CLVD ordered eigenvalue vector $\mathbf{M}^{*}=$ $(1,-1 / 2,-1 / 2)^{T}$ will give the following:

$$
\left[\begin{array}{l}
1 \\
-1 / 2 \\
-1 / 2
\end{array}\right]=\frac{3}{4}\left[\begin{array}{l}
1 \\
0 \\
-1
\end{array}\right]+\frac{1}{4}\left[\begin{array}{l}
1 \\
-2 \\
1
\end{array}\right]=M_{\mathrm{DC}}^{E} E_{\mathrm{DC}}^{E}+M_{\mathrm{CLVD}}^{E} E_{\mathrm{CLVD}}^{E}
$$

Although the Euclidean decomposition equation is exact and free from equality inconsistencies, such as Eq. 15, there are some other minor inconsistencies. (1) The unity problem: Because the input eigenvalue vector is a pure CLVD, the coefficient of the CLVD should be one instead of $1 / 4$. (2) The flip basis problem: There is an inconsistency between the input vector, which has a positive major element, and the corresponding basis, which has a negative major element, $(1,-2,1)^{T}$. One may naively think this inconsistency can be solved using the correct CLVD basis vector. Again, this will work, but it will inevitably introduce a new inconsistency. Without the flip basis, the decomposition sign of a pure CLVD $(1,-1 / 2,-1 / 2)^{T}$ will be wrong. Apparently, an artificial flip of the sign of the CLVD basis $E_{\mathrm{CLVD}}^{E}$ is essential to achieve the correct sign of the CLVD coefficient.

Furthermore, running the above pure CLVD example in the standard method will give us a reasonable answer,

$\left[\begin{array}{l}1 \\ -1 / 2 \\ -1 / 2\end{array}\right]=1\left[\begin{array}{c}1 \\ -1 / 2 \\ -1 / 2\end{array}\right]=M_{\mathrm{CLVD}}^{S} E_{\mathrm{CLVD}}^{S}$.

To summarize, we have demonstrated the inconsistencies in the two families of methods via two examples. The standard family appears to have equality inconsistency in the type II cases. The Euclidean family appears to have pure CLVD unity inconsistency and flip basis inconsistency.

\subsection{In search of an overall consistent decomposition method}

Although minor inconsistencies exist in both families of methods, one may still argue that these inconsistencies are trivial and can be addressed by simply reinterpreting the results. For example, in the standard method, one can ignore the equality issue and use only the coefficient $M_{\mathrm{CLVD}}^{S}$. In the Euclidean method, one can reinterpret the $M_{\mathrm{CLVD}}^{E}=1 / 4$ as the pure CLVD. For the flip basis problem, one can either flip the signs of the positive CLVD and negative CLVD in the scale plot (Chapman and Leaney 2012) or simply flip the sign of the CLVD basis (Tape and Tape 2012a) to address those inconsistencies. We argue the opposite. First, these minor inconsistencies existing at the framework level do not mean that they are not real. These inconsistencies indicate that there is serious tension between the results of the two families of methods and reasonable outcomes. A reconciliation is needed. Second, using the proposed new method, we can obtain insight into previous methods from a different, sometimes higher perspective. We could even examine the current methods in a more generalized fashion to evaluate how good the current methods work. Third, by requiring consistency, the resultant method may sometimes gain unexpected advantages 
over the old methods in regions outside the inconsistencies. After knowing all the inconsistencies of the previous methods, one may ask if it is possible to attain an overall consistent method, a method that avoids all the inconsistencies without producing any new inconsistencies, a method that can also maintain most of the good features in the existing methods.

In this paper, we prove that it is indeed possible to achieve that. The proof is done by designing a new decomposition method that solves all of the inconsistencies as an example. We propose such a method, called generalized orthonormal moment tensor decomposition (GOMTD). GOMTD inherits the orthogonality feature from the Euclidean family while possessing correct pure CLVD coefficients from the standard family of methods. The key insight in building GOMTD comes from the observation that the eigenvalue preordering process is the source of all the troubles, and it will suppress some orientational information in the moment tensors. A pure CLVD with its positive direction oriented toward the north is very different from a pure CLVD with its positive direction oriented to the east. However, after the eigenvalue preordering process, the two CLVDs will become indistinguishable. In GOMTD, we replace the eigenvalue preordering process with three sets of generalized bases and a selection rule. In doing so, GOMTD calculates coefficients from all orientations and keeps track of the orientation information.

Our contributions are as follows:

- We develop GOMTD and document its calculation procedure (Section 2).

- We demonstrate how GOMTD can successfully handle all of the abovementioned inconsistencies (Section 3.1).

- We explored some behaviors of GOMTD through scale factor plot comparison with previous methods (Sections 3.2, 3.3, 3.4 and 3.5).

- We discuss the behavior of GOMTD and other methods under a fixed-scale factor ratio case (Section 3.7).

- We discuss the role GOMTD can play in the usage of CLVD scale factors and in the usage of an orientation index (Sections 3.6 and 3.9).

- We show how to extend GOMTD and discuss the limitations of GOMTD (Sections 3.11 and 3.12).

\section{Generalized orthonormal moment tensor decomposition}

Figure 1 summarizes the entire GOMTD algorithm. We start with a 3-by-3 moment tensor matrix $\mathbf{M}$.

\subsection{Diagonalization}

The first step is to diagonalize the moment tensor matrix. In GOMTD, we do not need to preorder the three eigenvalues. Instead, we directly use the unordered eigenvalues in vector form, that is,

$\mathbf{M}=\left[\begin{array}{l}M_{1} \\ M_{2} \\ M_{3}\end{array}\right]$,

which will be called an eigenvalue vector hereinafter.

\subsection{Calculating three sets of coefficients}

Three orthonormal bases exist with which to decompose the eigenvalue vector into three fundamental components, namely, the ISO component, the DC component, and the CLVD component. The first orthonormal basis is

$\hat{E}_{\mathrm{ISO}}^{(1)}=\frac{1}{\sqrt{3}}\left[\begin{array}{l}1 \\ 1 \\ 1\end{array}\right], \hat{E}_{\mathrm{DC}}^{(1)}=\frac{1}{\sqrt{2}}\left[\begin{array}{l}0 \\ 1 \\ -1\end{array}\right], \hat{E}_{\mathrm{CLVD}}^{(1)}=\frac{1}{\sqrt{6}}\left[\begin{array}{c}2 \\ -1 \\ -1\end{array}\right]$.

The second orthonormal basis is

$\hat{E}_{\mathrm{ISO}}^{(2)}=\frac{1}{\sqrt{3}}\left[\begin{array}{l}1 \\ 1 \\ 1\end{array}\right], \hat{E}_{\mathrm{DC}}^{(2)}=\frac{1}{\sqrt{2}}\left[\begin{array}{l}1 \\ 0 \\ -1\end{array}\right], \hat{E}_{\mathrm{CLVD}}^{(2)}=\frac{1}{\sqrt{6}}\left[\begin{array}{l}-1 \\ 2 \\ -1\end{array}\right]$.

Finally, the third orthonormal basis is

$\hat{E}_{\mathrm{ISO}}^{(3)}=\frac{1}{\sqrt{3}}\left[\begin{array}{l}1 \\ 1 \\ 1\end{array}\right], \hat{E}_{\mathrm{DC}}^{(3)}=\frac{1}{\sqrt{2}}\left[\begin{array}{l}1 \\ -1 \\ 0\end{array}\right], \hat{E}_{\mathrm{CLVD}}^{(3)}=\frac{1}{\sqrt{6}}\left[\begin{array}{l}-1 \\ -1 \\ 2\end{array}\right]$. 


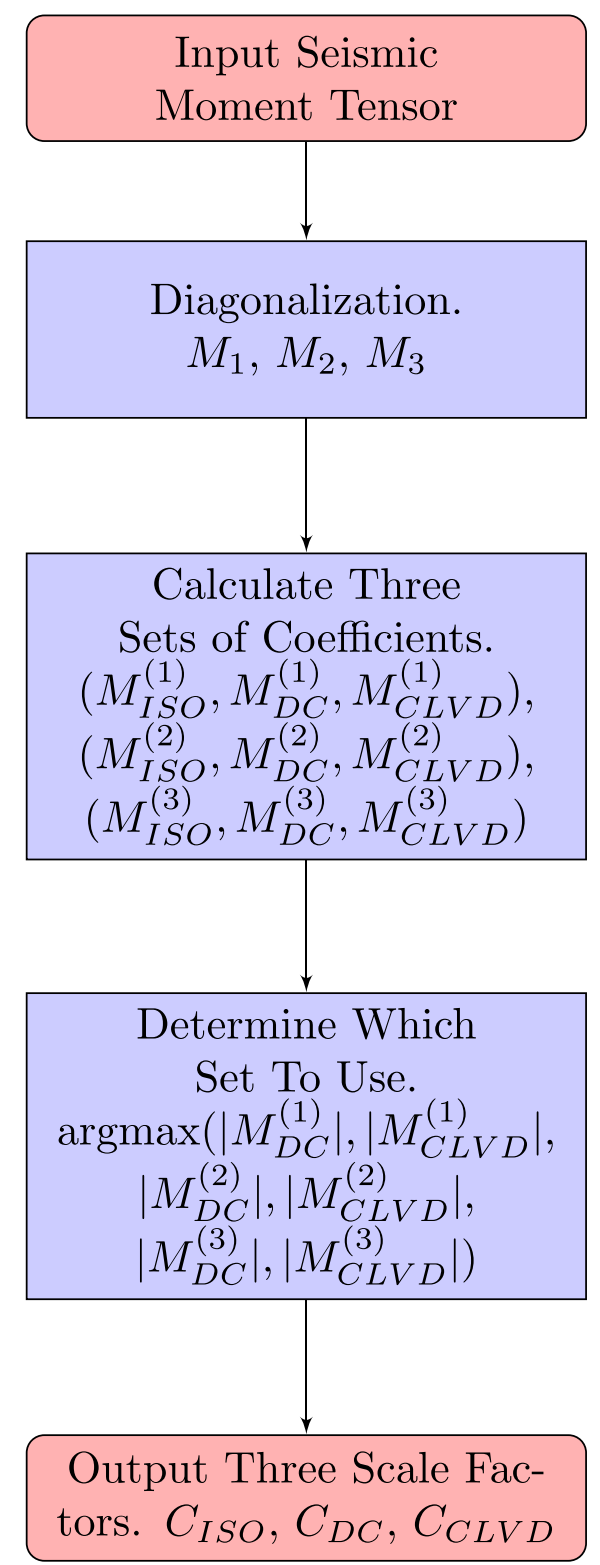

Fig. 1 Flowchart of the proposed moment decomposition method

The coefficients are simply projections along these bases. For example, the coefficients of the first basis are

$$
\begin{aligned}
M_{\mathrm{ISO}}^{(1)} & =\mathbf{M} \cdot \hat{E}_{\mathrm{ISO}}^{(1)}, \\
& =\frac{1}{\sqrt{3}}\left(M_{1}+M_{2}+M_{3}\right), \\
M_{\mathrm{DC}}^{(1)} & =\mathbf{M} \cdot \hat{E}_{\mathrm{DC}}^{(1)}, \\
& =\frac{1}{\sqrt{2}}\left(M_{2}-M_{3}\right),
\end{aligned}
$$

$$
\begin{aligned}
M_{\mathrm{CLVD}}^{(1)} & =\mathbf{M} \cdot \hat{E}_{\mathrm{CLVD}}^{(1)}, \\
& =\frac{1}{\sqrt{6}}\left(2 M_{1}-M_{2}-M_{3}\right) .
\end{aligned}
$$

Similar calculations can be carried out for the second and third bases.

\subsection{Determining which set to use}

Once all three sets of coefficients are available, GOMTD will decide which set to use. First, we 
observe that the ISO coefficients are the same for all three bases, that is, $M_{\mathrm{ISO}}^{(1)}=M_{\mathrm{ISO}}^{(2)}=M_{\mathrm{ISO}}^{(3)}$. Because the ISO coefficients are independent of the chosen basis, we do not need to include them within the selection rules. Consequently, we have six coefficients, two for each basis. To account for the pure CLVD unity problem, we design the GOMTD selection rules to find the basis containing the six coefficients with the largest amplitudes,

$\underset{n}{\operatorname{argmax}}\left\{\left|M_{\mathrm{DC}}^{(n)}\right|,\left|M_{\mathrm{CLVD}}^{(n)}\right|\right\}$,

where the support is $n=\{1,2,3\}$. After the selection rule, there will be two types of output. One is the index of the bases used in the algorithm, which corresponds to the spatial orientation of the moment tensor. The other contains the coefficients of the given basis. They are similar to the Euclidean type coefficients except for the areas with inconsistencies.

\subsection{Three output scale factors}

Once the basis to use is determined, the corresponding coefficients are calculated. GOMTD will then determine the scalar moment and calculate the scale factors. The chosen coefficients are now $M_{\mathrm{ISO}}, M_{\mathrm{DC}}$, and $M_{\mathrm{CLVD}}$. Note that the basis indicators have been suppressed in the equation for simplicity. The scalar moment is defined as

$M_{0}=\sqrt{M_{1}^{2}+M_{2}^{2}+M_{2}^{3}}=\sqrt{M_{\mathrm{ISO}}^{2}+M_{\mathrm{DC}}^{2}+M_{\mathrm{CLVD}}^{3}}$.

The three scale factors are expressed as

$$
C_{\mathrm{ISO}}=\frac{M_{\mathrm{ISO}}}{M_{0}}, C_{\mathrm{DC}}=\frac{M_{\mathrm{DC}}}{M_{0}}, C_{\mathrm{CLVD}}=\frac{M_{\mathrm{CLVD}}}{M_{0}},
$$

where

$$
\begin{aligned}
\mathbf{M} & =M_{\mathrm{ISO}} \hat{E}_{\mathrm{ISO}}+M_{\mathrm{DC}} \hat{E}_{\mathrm{DC}}+M_{\mathrm{CLVD}} \hat{E}_{\mathrm{CLVD}}, \\
& =M_{0}\left(C_{\mathrm{ISO}} \hat{E}_{\mathrm{ISO}}+C_{\mathrm{DC}} \hat{E}_{\mathrm{DC}}+C_{\mathrm{CLVD}} \hat{E}_{\mathrm{CLVD}}\right) .
\end{aligned}
$$

Note that although the above expression is very similar to the expressions of the standard method and the Euclidean method, the meaning is quite different. GOMTD, which contains an orientation index, can restore the original eigenvalue vectors $(\mathbf{M})$, while the other two methods can only restore the ordered eigenvalue vectors. Moreover, these methods exhibit some orientation loss. Additionally, note that the above decomposition can be written in diagonal matrix form. When using the spectral decomposition theorem, those diagonal matrices can be restored into a matrix form that decomposes the raw moment tensor. The pure DC moment tensor in matrix form can give the $\mathrm{P}, \mathrm{T}$, and $\mathrm{N}$ axes. The calculation of restoration to matrix form is more natural in GOMTD and reflects the advantage of not suppressing orientational information.

Figure 2 shows the source-type diagram of GOMTD. It is visualized by the scale factor plot of $C_{I S O}$ and $C_{C L V D}$, both derived from the proposed method. In the scale factor plot, the possible values of the ISO and CLVD scale factors are between -1 and 1 rather than following the shape of a diamond as in the standard method or the shape of a lune as in the Euclidean method. Later, the permitted region of scale factors will be shown to consist of a lune and a circular boundary.

\section{Discussion}

\subsection{GOMTD can handle the inconsistencies}

As an example, we demonstrate that GOMTD can correctly handle the two minor inconsistencies presented in the introduction section. First, we reconsider the case of a pure CLVD, $(1 / 2,1 / 2,-1)^{T}$, as an illustration of the equality inconsistency. Using the proposed method, the six corresponding coefficients are as follows:

$M_{\mathrm{DC}}^{(1)}=1.061, \quad M_{\mathrm{CLVD}}^{(1)}=0.612$

$M_{\mathrm{DC}}^{(2)}=1.061, \quad M_{\mathrm{CLVD}}^{(2)}=0.612$

$M_{\mathrm{DC}}^{(3)}=0, \quad M_{\mathrm{CLVD}}^{(3)}=-1.225$.

The coefficient with the largest amplitude is $M_{\mathrm{CLVD}}^{(3)}$. Therefore, the chosen basis is 3 . The proposed decomposition is expressed as

$\left[\begin{array}{c}1 / 2 \\ 1 / 2 \\ -1\end{array}\right]=\frac{-3}{\sqrt{6}}\left[\begin{array}{c}-1 / \sqrt{6} \\ -1 / \sqrt{6} \\ 2 / \sqrt{6}\end{array}\right]=M_{\mathrm{CLVD}}^{(3)} \hat{E}_{\mathrm{CLVD}}^{(3)} \cdot$ 


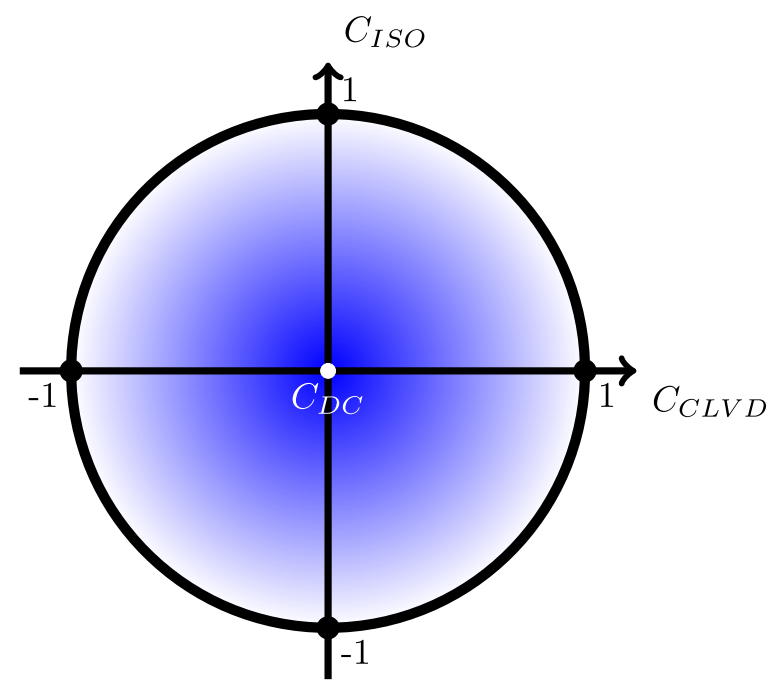

Fig. $2 C_{\text {CLVD }}-C_{\text {ISO }}$ circular plot of the proposed moment decomposition method. The shading intensity corresponds to the scale factor of the DC component

Moreover, the corresponding scale factor is

$C_{\mathrm{CLVD}}=\frac{M_{\mathrm{CLVD}}^{(3)}}{M_{0}}=\frac{\frac{-3}{\sqrt{6}}}{\sqrt{3 / 2}}=-1$

Note that both the scale factor and the sign of the eigenbasis have the correct signs now. Therefore, the proposed method can handle the equality inconsistency in the standard method.

Next, we look into the case of the pure CLVD, $(1,-1 / 2,-1 / 2)^{T}$, as an illustration of the pure CLVD unity inconsistency. In this case, in which a basis of 1 is chosen for the proposed method, the decomposition of the proposed method is expressed as follows:

$$
\left[\begin{array}{l}
1 \\
-1 / 2 \\
-1 / 2
\end{array}\right]=\frac{3}{\sqrt{6}}\left[\begin{array}{l}
2 / \sqrt{6} \\
-1 / \sqrt{6} \\
-1 / \sqrt{6}
\end{array}\right]=M_{\mathrm{CLVD}}^{(1)} \hat{E}_{\mathrm{CLVD}}^{(1)}
$$

The only nonzero scale factor is

$C_{\mathrm{CLVD}}=\frac{M_{\mathrm{CLVD}}^{(1)}}{M_{0}}=\frac{\frac{3}{\sqrt{6}}}{\sqrt{3 / 2}}=1$

Therefore, the unity of the pure CLVD scale factor is preserved. Moreover, there is no flip sign eigenbasis inconsistency in GOMTD, as the sign of the eigenbasis basis $\hat{E}_{\mathrm{CLVD}}^{(1)} \propto(2,-1,-1)^{T}$ is in accordance with the input eigenvalue vector. In GOMTD, there is no need to impose an artificial sign flip on the CLVD basis or flip the scale plot. Everything follows through naturally.

\subsection{Scale factor plots for random moment tensors}

We wish to demonstrate the distribution of GOMTD. Figure 3 shows a scale factor $C_{\mathrm{CLVD}}-C_{\text {ISO }}$ plot comparison of GOMTD, the standard method, and the Euclidean method using 5000 data points from a randomly generated eigenvalue vector, that is,

$$
\left[\begin{array}{c}
\text { Uniform }(-1.0,1.0) \\
\text { Uniform }(-1.0,1.0) \\
\text { Uniform }(-1.0,1.0)
\end{array}\right] \text {. }
$$

The scale factor plot shows that GOMTD has a lune distribution and a circular boundary. It attracts data points along the circumference of the circular boundary, whereas the standard method and the Euclidean method show a somewhat uniform distribution and follow their own permitted region, which is diamond shaped for the standard method and lune shaped for the Euclidean method. A lengthy discussion of the probability densities of the two previously developed methods can be found in previous studies (Chapman 2019; Tape and Tape 2012b).

\subsection{Scale factor plots when the moment tensor surrounds a pure ISO vector}

Figure 4 shows a scale factor $C_{\mathrm{CLVD}}-C_{\mathrm{ISO}}$ plot comparison of GOMTD, the standard method, and the Euclidean method using 5000 data points from the 

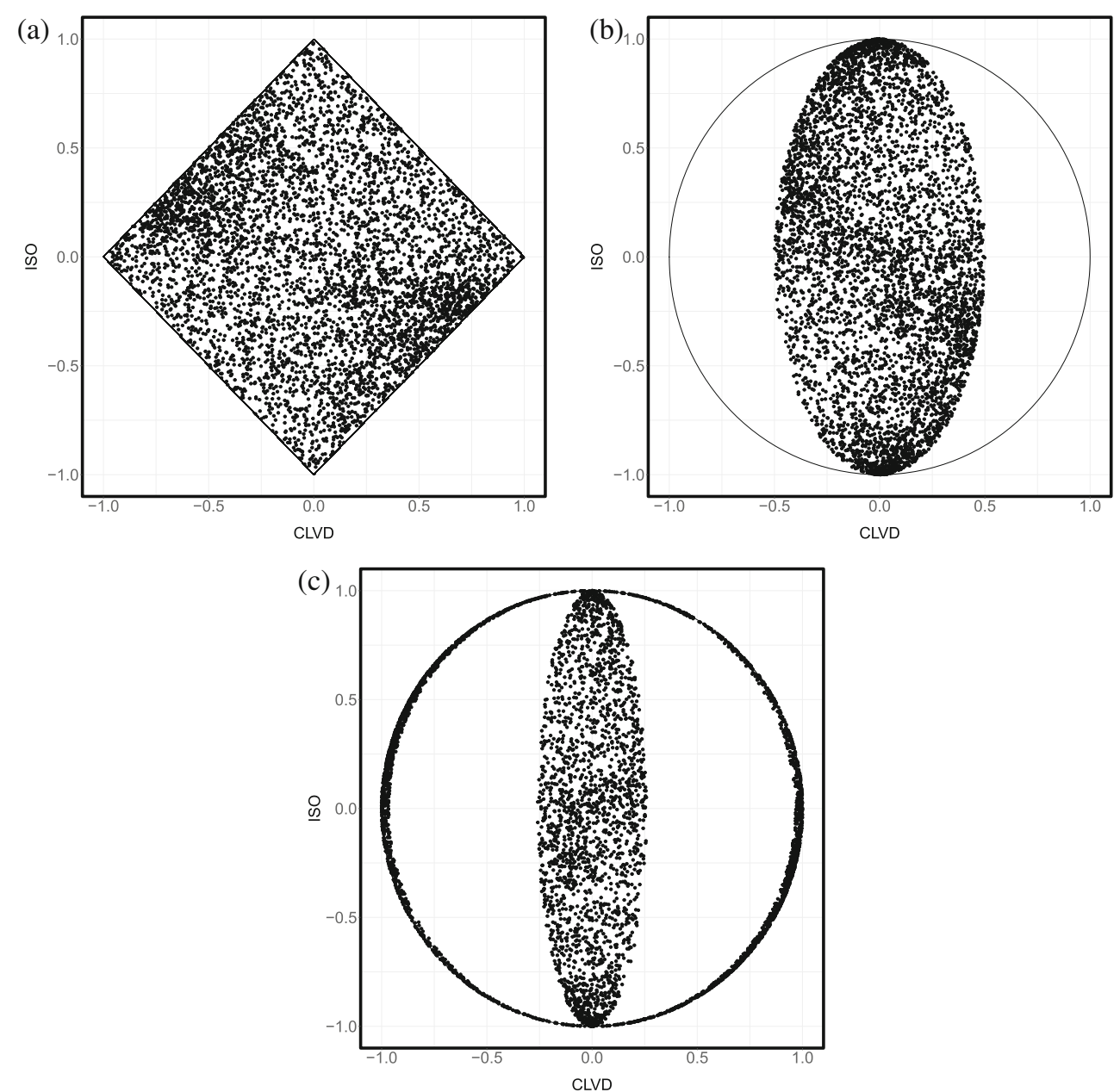

Fig. 3 Scale factor $C_{\mathrm{CLVD}}-C_{\text {ISO }}$ plots of simulations of random moment tensors. a Standard method. b Euclidean method. $\mathbf{c}$ Proposed method

generated eigenvalue vector around a pure ISO vector with some random noise, that is,

$$
\left[\begin{array}{c}
1+\text { Uniform }(-0.25,0.25) \\
1+\operatorname{Uniform}(-0.25,0.25) \\
1+\operatorname{Uniform}(-0.25,0.25)
\end{array}\right]
$$

The results show that GOMTD attracts pure ISO vectors with some deviation toward the pure ISO poles. A similar situation also happens with the Euclidean method but with less concentration around the circumference. Both the proposed method and the Euclidean method outperform the standard method in this case. Note that the choice of $(1,1,1)$ is without loss of generality.

\subsection{Scale factor plots when the moment tensor surrounds a pure DC vector}

Figure 5 shows a scale factor $C_{\mathrm{CLVD}}-C_{\mathrm{ISO}}$ plot comparison of GOMTD, the standard method, and the Euclidean method using 5000 data points from the generated eigenvalue vector around one pure DC vector with some random noise, that is,

$$
\left[\begin{array}{c}
1+\text { Uniform }(-0.25,0.25) \\
0+\operatorname{Uniform}(-0.25,0.25) \\
-1+\operatorname{Uniform}(-0.25,0.25)
\end{array}\right] \text {. }
$$

The results show that GOMTD, along with the Euclidean method, has a smaller deviation than the 

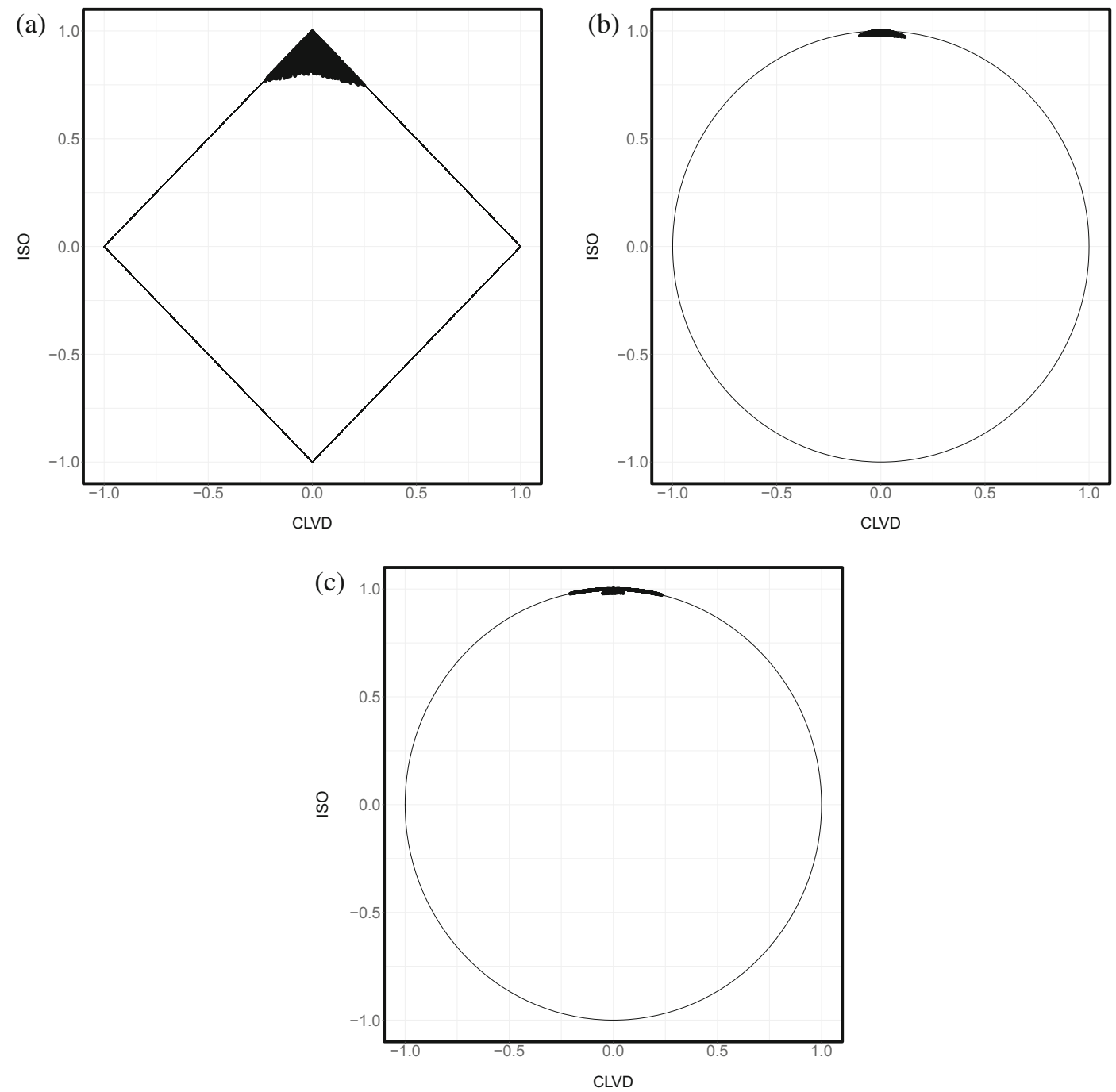

Fig. 4 Scale factor $C_{\mathrm{CLVD}}-C_{\mathrm{ISO}}$ plots of simulations of random moment tensors around a pure ISO mechanical type. a Standard method. b Euclidean method. c Proposed method

standard method. A similar result will prevail when the pure DC eigenvalue vector is along another orientation, say $(0,1,-1)$. However, there are some outlier points located near the boundary of the circle for the proposed method. Further examination reveals that these points are essentially ambiguous between the DC and CLVD components. For example, one of the points located around the left boundary is $\mathbf{M}=(-1.149,0.247,0.757)$, which will be considered a CLVD component, that is, $-(2,-1,-1)$, in the proposed method.

Another important point we can observe is the mirror symmetry between GOMTD and the Euclidean method. There is almost a one-to-one correspondence between the points of the two methods, only off by the sign of the CLVD. The mirror symmetry can be explained by the CLVD basis sign flip in the Euclidean method. The CLVD basis of the two methods is off by a minus sign.

Examining all of the simulated data points, we can further observe that there is an extra minus sign problem in the standard method as well. This is due to the preordering process and will eventually be restored to the correct sign when the CLVD factor becomes large enough to trigger an order flip in the preordering process. 

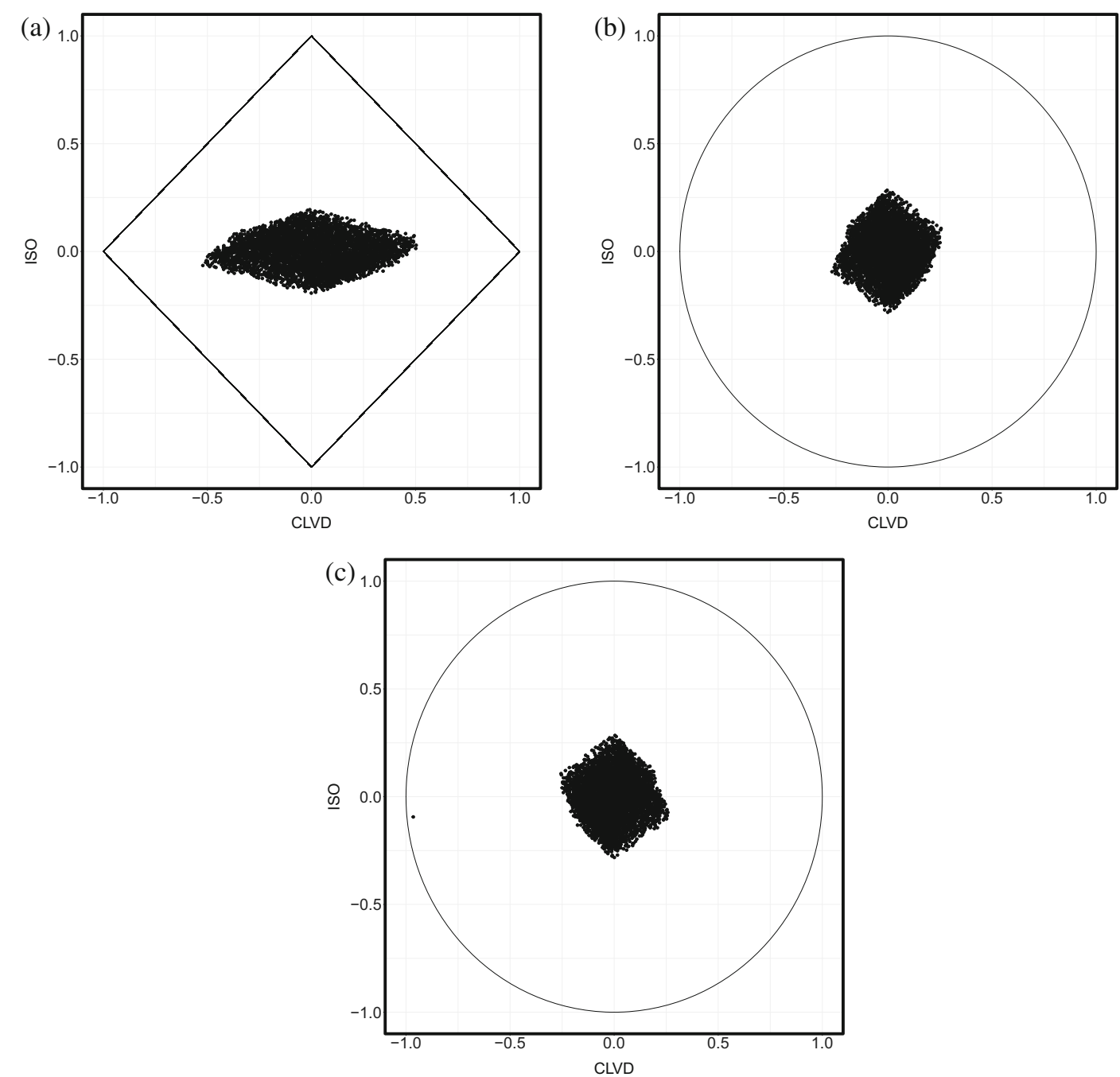

Fig. 5 Scale factor $C_{\mathrm{CLVD}}-C_{\mathrm{ISO}}$ plots of simulations of random moment tensors around a pure DC mechanical type. a Standard method. b Euclidean method. c Proposed method

To summarize the above, there is a sign problem when the CLVD is disentangled from the DCdominated case. To illustrate the problem, we consider a pure DC with some small positive CLVD,

$$
\frac{1}{\sqrt{2}}\left[\begin{array}{l}
1 \\
0 \\
-1
\end{array}\right]+0.1 \frac{1}{\sqrt{6}}\left[\begin{array}{l}
-1 \\
2 \\
-1
\end{array}\right] \rightarrow\left\{\begin{array}{l}
\text { (ISO, DC, CLVD) } \\
\text { the proposed method, } \\
(0.0,0.995,0.10) \\
\text { the standard method, } \\
(0.0,0.782,-0.22) \\
\text { the Euclidean method, } \\
(0.0,0.995,-0.10)
\end{array}\right.
$$

Note that only GOMTD will disentangle obtaining a correct sign of the CLVD scale factor $(+0.10)$. The other two methods will disentangle negative CLVD scale factors $(-0.22,-0.10)$ until the eigenvalue vector becomes a CLVD-dominated case, which will induce a flip in the preordering process.

\subsection{Scale factor plots when the moment tensor surrounds a pure CLVD vector}

Figure 6 shows a scale factor $C_{\mathrm{CLVD}}-C_{\mathrm{ISO}}$ plot comparison of GOMTD, the standard method, and the Euclidean method using 5000 data points from the generated eigenvalue vector around a pure CLVD 
vector with some random noise, that is,

$$
\left[\begin{array}{c}
2+\operatorname{Uniform}(-0.25,0.25) \\
-1+\operatorname{Uniform}(-0.25,0.25) \\
-1+\operatorname{Uniform}(-0.25,0.25)
\end{array}\right] \text {. }
$$

The results show that GOMTD attracts data points around the CLVD toward the CLVD poles. GOMTD outperforms both the standard method and the Euclidean method in this case. A similar result will prevail when the pure CLVD eigenvalue vector is along another orientation, e.g., $(-1,2,-1)$. Such a unique denoising feature can further stabilize the resulting CLVD scale factors under some noise perturbation.

\subsection{The issue of the CLVD scale factor}

In several previous studies (Vavryčuk and Hrubcová 2017; Kagan 2003), researchers examined various seismic data and claimed that the CLVD components were not very accurate. Moreover, the authors of a recent study (Yu et al. 2018) claimed that the CLVD scale factor (i.e., the percentage) is not a good statistical quantity due to its sensitivity to noise.

Regarding the issue of the CLVD, we believe that GOMTD can be helpful. First, GOMTD is free from the sign problem around the DC, and it will not add more burden to the already inaccurate CLVD data. Second, GOMTD has a unique denoising feature, meaning that it is possible to stabilize noisy CLVD data.

\subsection{Scale factor plots when the ratio of $C_{I S O} / C_{C L V D}$ is fixed}

Vavryčuk (2001) suggested that for pure tensile and shear-tensile sources, the ISO/CLVD ratio depends solely on $V_{P} / V_{S}$. We compare the scale factor plots when the ratio of $C_{I S O} / C_{C L V D}$ is fixed. The test eigenvalue vector is as follows:

$E_{I S O}^{S}+2 E_{C L V D}^{S}+C E_{D C}^{S}$,

where $C$ is a constant that controls the weight of the DC component. We examine two cases: a large $C$ case and a small $C$ case. Figure 7 shows the scale factor plots when $C$ is large. One can notice that GOMTD shows a straight line with some circular components but opposite in slope with the Euclidean method. It is understandable because there is a base flip between the two. GOMTD does not possess a good linear relation in the large $C$ case, as with the previous methods. Figure 8 shows the scale factor plots when $C$ is small. In this case, both the standard method and the Euclidean method feature a straight line. GOMTD shows an area of points around the outer circle. This is due to the pole attraction effect. Although it seems that the standard method can best reconstruct the linear relation from Eq. 46, we doubt the validity of adding three bases that are not orthogonal to each other. Note that a similar linear relation construct by orthogonal bases, either the Euclidean method or GOMTD, will result in a messy reconstruction for all three methods.

\subsection{Scale factor plots of Geyser geothermal events}

Figure 9 depicts a scale factor plot comparison among GOMTD, the standard method, and the Euclidean method using 53 focal mechanisms located at the Geysers Geothermal Field, California. The detailed focal mechanism dataset can be found in Table 2 of Boyd et al. (2015). Figure 9(a) reproduces the results of Figure 2(a) of Boyd et al. (2015). Overall, all three methods present similar results, but GOMTD automatically separates the events into three groups, one dominated by DC events, one dominated by positive CLVD events, and one dominated by negative CLVD events. These findings demonstrate that GOMTD is suitable for cluster analysis. In this dataset, the CLVD-dominated groups appear in the standard method and Euclidean method plots, albeit not as prominently as in the GOMTD plot.

\subsection{Orientation index of the Orthonormal bases}

In the middle of the moment tensor decomposition algorithm of the proposed method, we decide which set of orthonormal bases to use, namely, $\hat{E}^{(1)}, \hat{E}^{(2)}$, or $\hat{E}^{(3)}$. The chosen index of orthonormal bases for each seismic event can provide additional orientation information that would otherwise be suppressed by the eigenvalue preordering process. In practice, one can identify the dominant orthonormal basis of one area and associate it with the geological setting. In addition, one can easily classify seismic events into different groups by their indices of bases. 

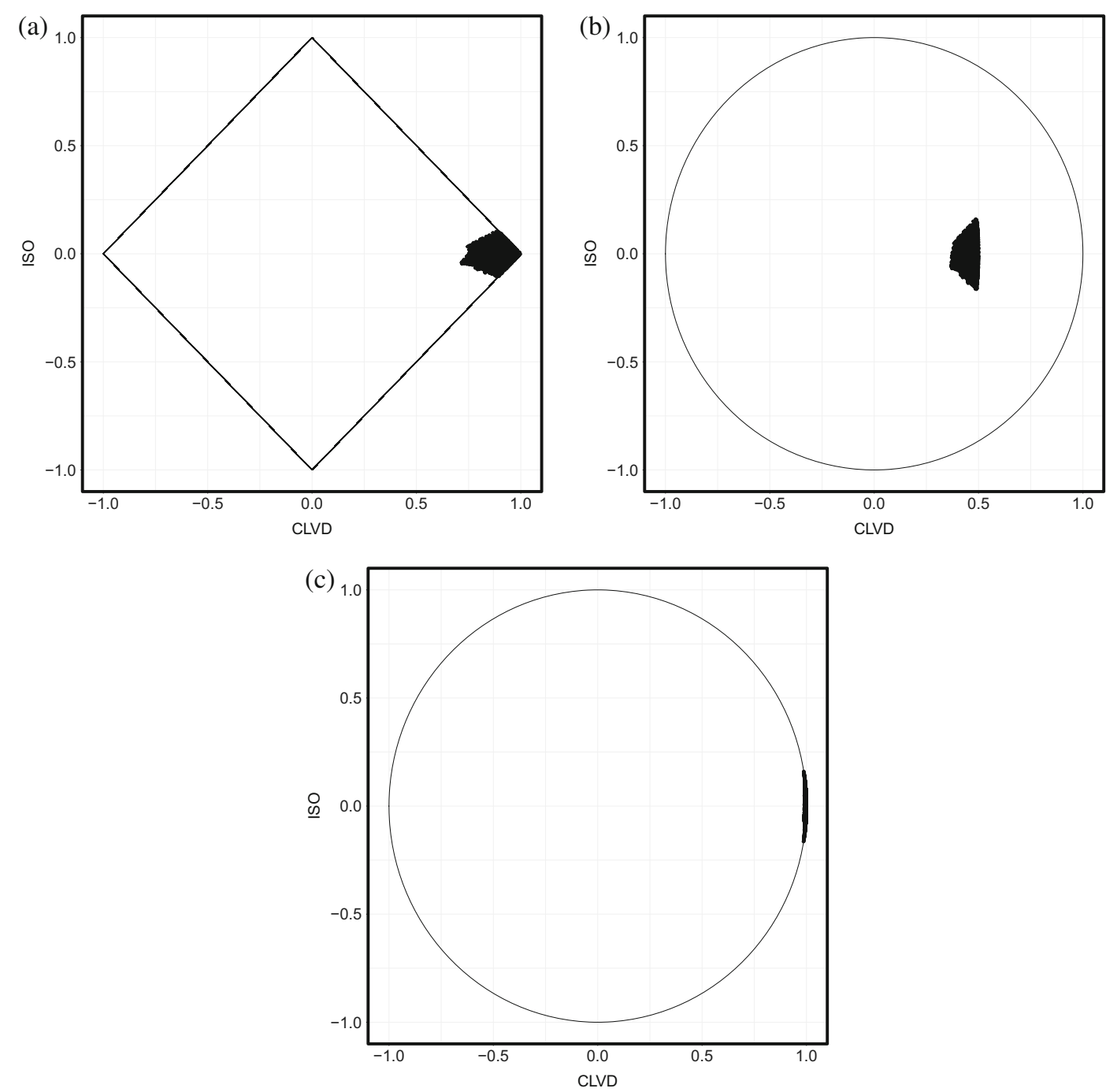

Fig. 6 Scale factor $C_{\mathrm{CLVD}}-C_{\mathrm{ISO}}$ plots of simulations of random moment tensors around a pure CLVD mechanical type. a Standard method. b Euclidean method. c Proposed method

\subsection{Order of growth analysis of the GOMTD algorithm}

We examine the order of growth of our proposed algorithm following the notation from Cormen et al. (2009). Considering that the inputs are $n$ sets of focal mechanisms, the worst running time of the proposed method is $\Theta(n)$. That is, the running time grows in proportion with the number of input events. Similar analysis shows that the running times for the standard method and the Euclidean method are also $\Theta(n)$. That is, the speed of the proposed method is on par with those of the standard method and the Euclidean method, although the proposed method contains more instructions.

\subsection{GOMTD extension and its connection to the}

Euclidean method

We can extend the concept of selection rules by including artificial weights to adapt to the geological setting of the research area. The extension selection rule is as follows:

$$
\underset{n}{\operatorname{argmax}}\left\{\left|w_{\mathrm{DC}}^{(n)} M_{\mathrm{DC}}^{(n)}\right|,\left|w_{\mathrm{CLVD}}^{(n)} M_{\mathrm{CLVD}}^{(n)}\right|\right\},
$$



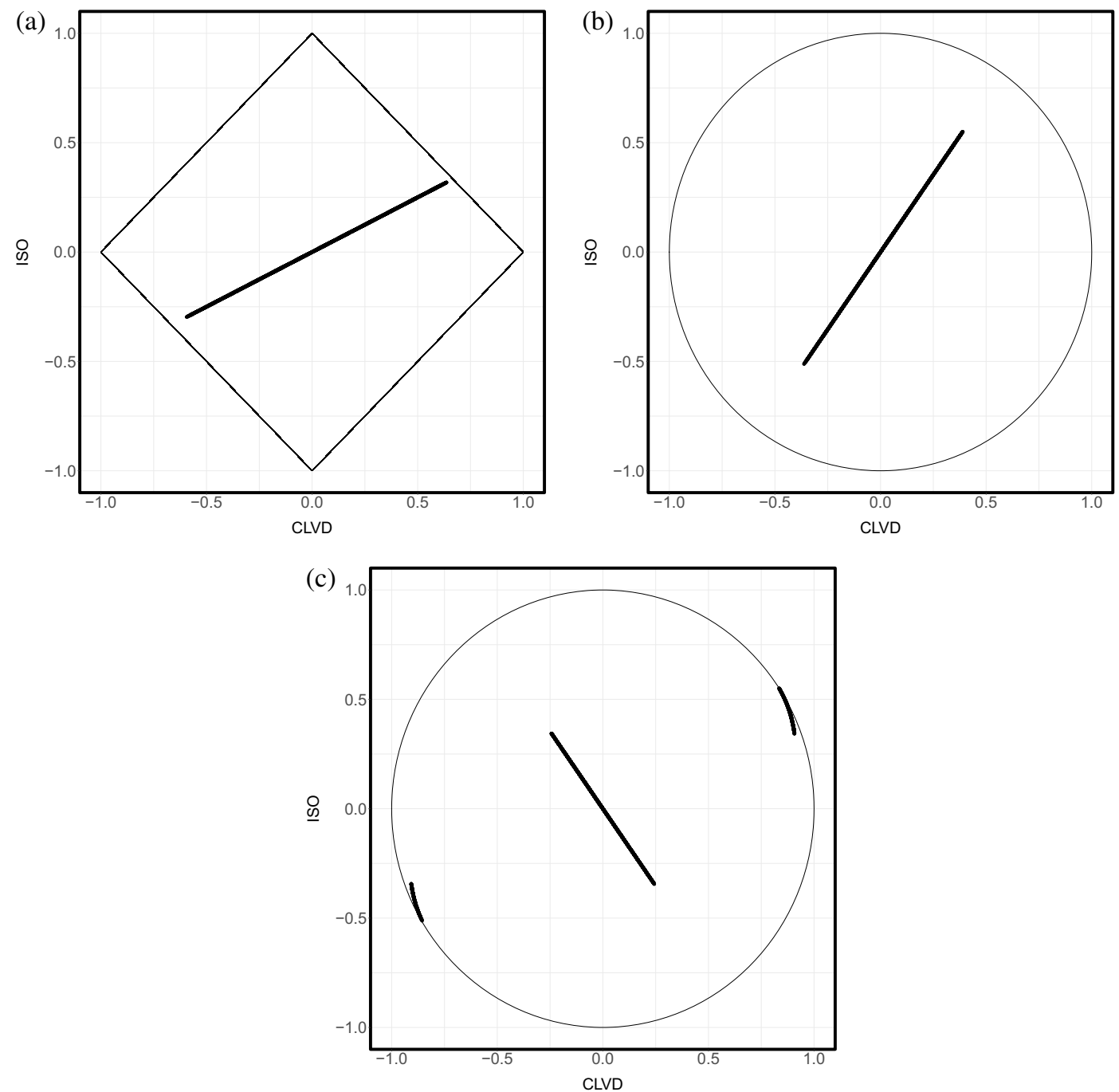

Fig. 7 Scale factor $C_{\mathrm{CLVD}}-C_{\text {ISO }}$ plots of simulations of a fixed-scale factor ratio with a large DC component. a Standard method. b Euclidean method. c Proposed method

where the $w$ terms represent different weights. When $w_{\mathrm{DC}}^{(2)}=w_{\mathrm{CLVD}}^{(2)}=1$ and the rest of the weights are set to zero, the moment tensor decomposition will be restored to the original Euclidean decomposition method (off by the minus sign in the CLVD basis, of course). The weights are important tools to allow the user to describe the possible orientation of the research area. For example, one can increase the weight of index 1 when the average moment tensor decompositions prefer the orientation of index 1. Alternatively, one could decrease the weight of index 2 when historical data show are less likely to exhibit such an orientation.

\subsection{Ambiguities and limitations}

Ambiguities usually arise when the dominant coefficients in two different bases are the same or nearly identical. It is also the cause of the transition area between the circular boundary and the lune in the GOMTD scale factor graph. It is true that the other methods do not have such transition area features. Although the transition area can be daunting at first, we believe it is actually not an unwanted feature. That is, the ambiguities are intrinsic (regardless of methods), and we believe it is better to show explicitly than sweep it under the rug. In practice, choosing a 

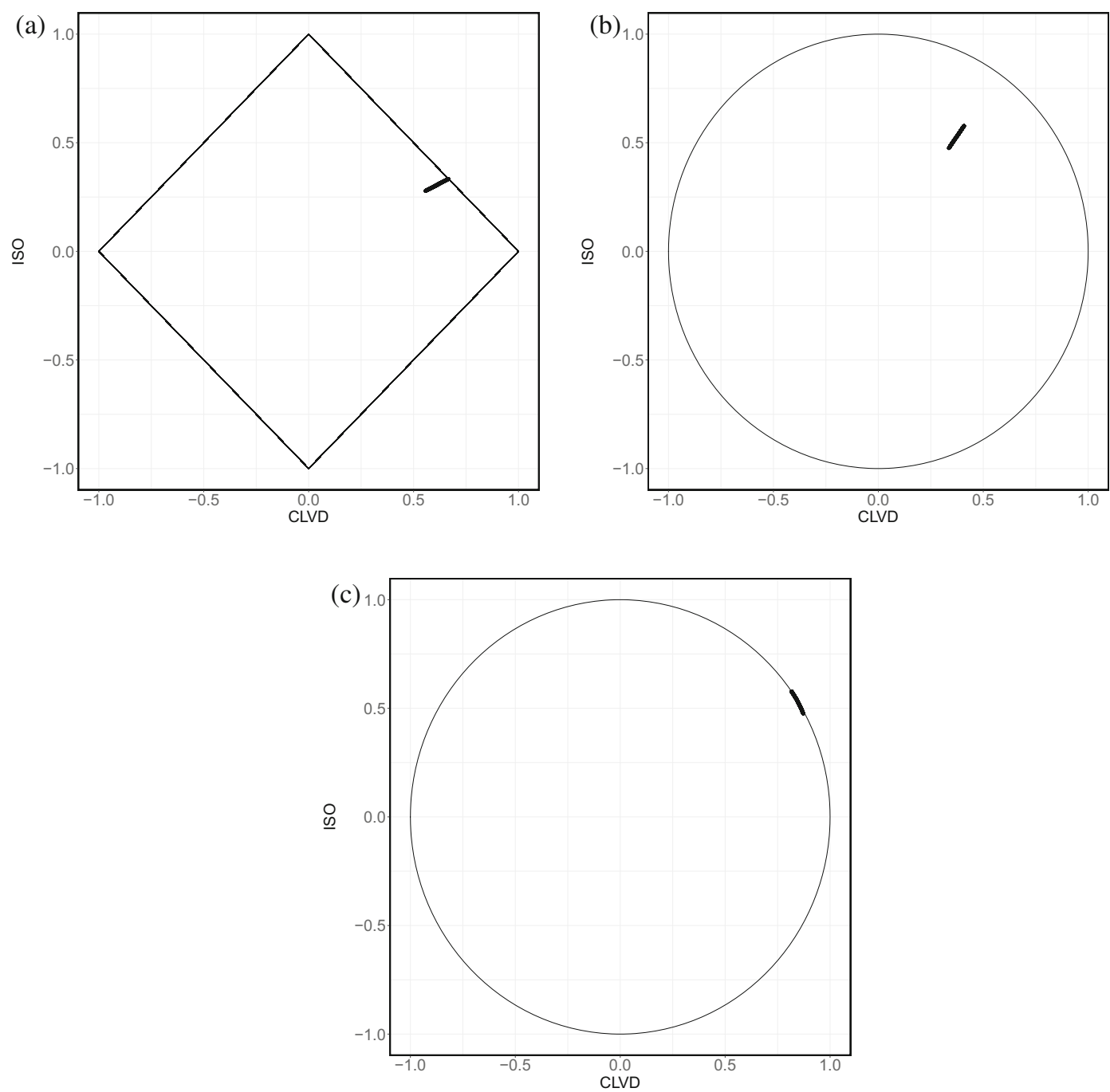

Fig. 8 Scale factor $C_{\mathrm{CLVD}}-C_{\mathrm{ISO}}$ plots of simulations of a fixed-scale factor ratio with a small DC component. a Standard method. b Euclidean method. c Proposed method

dominant basis by giving more weights in one area beforehand can significantly reduce these ambiguities.

\section{Conclusions}

This project starts with the observations of inconsistencies hidden in the current moment tensor decomposition methods, and all quick fixes introduce new inconsistencies. We demonstrate that it is possible to have a decomposition method free from those inconsistencies. We propose one such method called GOMTD, an overall consistent method that not only preserves the orthogonality of the bases but also addresses the inconsistencies of previous methods. Moreover, GOMTD can correctly disentangle the CLVD from the DC-dominated case. Furthermore, GOMTD tends to denoise the perturbation for ISO- and DC-dominated cases. In theoretical aspects, GOMTD is a reasonable alternative framework for moment tensor decomposition, and it can lead the way to inspire new methods. In practice, we believe that GOMTD will also be a helpful tool. The denoising feature, CLVD disentanglement ability, orientation index, and freedom to assign weights of GOMTD will be useful for future data-driven investigations, cluster analyses, and explorations, especially for extracting non-DC components. 

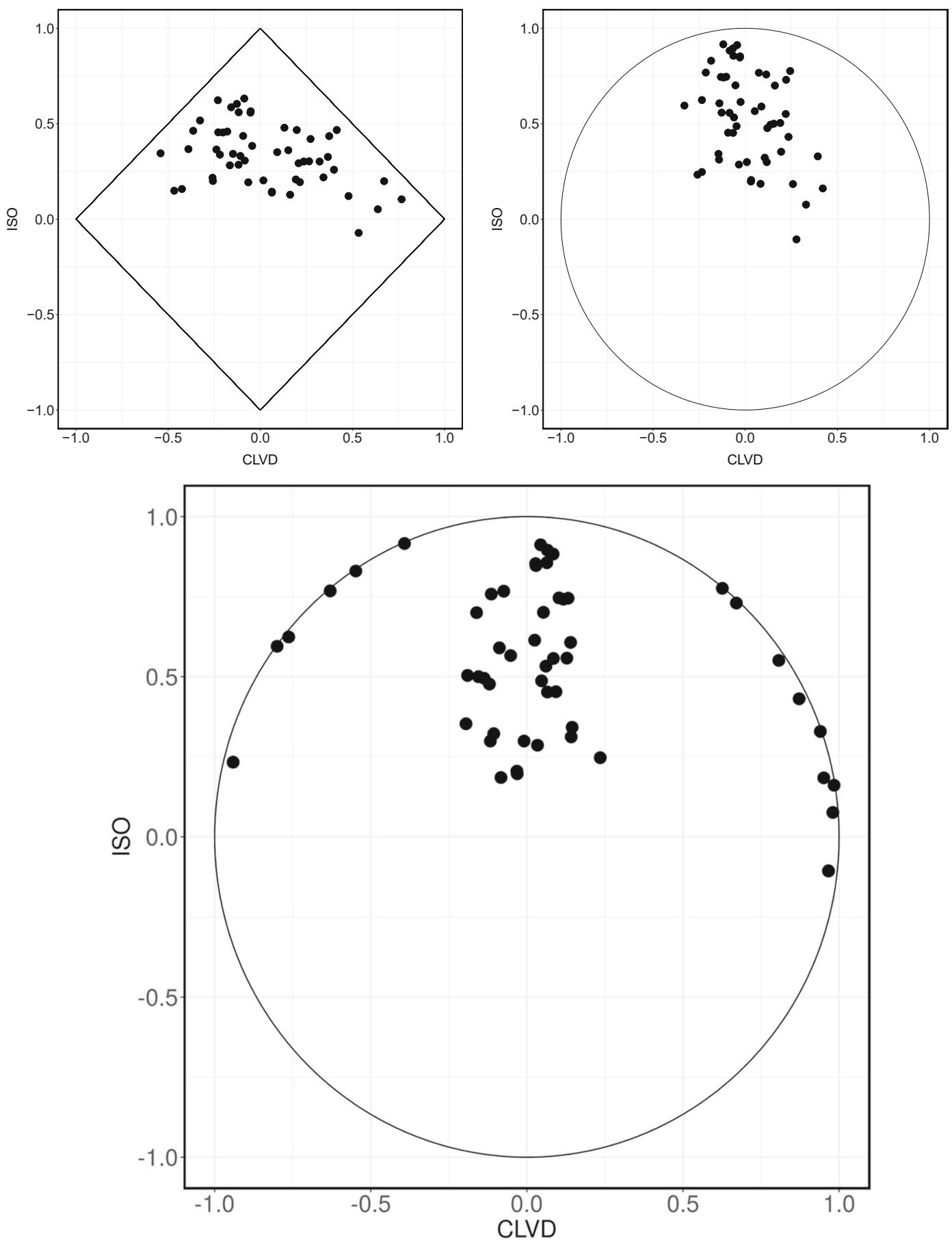

Fig. 9 Scale factor $C_{\mathrm{CLVD}}-C_{\text {ISO }}$ plots of 53 focal mechanisms at the Geysers Geothermal Field, California. a Standard method. b Euclidean method. c Proposed method

Funding Our work was supported by the Ministry of Science and Technology (MOST) of Taiwan under grant number 106-2116-M-002-019-MY3 and 109-2116-M-002-030- MY3. Our work was also supported by the Research Center of Future Earth of the National Taiwan University (NTU). This work was also financially supported by the NTU Research Center for Future Earth from The Featured Areas Research Center Program within the framework of the Higher Education Sprout Project by the Ministry of Education (MOE) in Taiwan. 
Author contributions T.C. developed the theoretical formalism and wrote the paper. Y.M. supervised the findings of this work. All authors discussed the results and contributed to the final manuscript.

Data availability The code used in this study will be on the author's GitHub. It will also be available by the authors upon request. All the Geyser focal mechanism data are listed in Boyd et al. (2015).

\section{Compliance with ethical standards}

Competing interests The authors declare that they have no competing interests.

Ethics approval and consent to participate Not applicable.

Open Access This article is licensed under a Creative Commons Attribution 4.0 International License, which permits use, sharing, adaptation, distribution and reproduction in any medium or format, as long as you give appropriate credit to the original author(s) and the source, provide a link to the Creative Commons licence, and indicate if changes were made. The images or other third party material in this article are included in the article's Creative Commons licence, unless indicated otherwise in a credit line to the material. If material is not included in the article's Creative Commons licence and your intended use is not permitted by statutory regulation or exceeds the permitted use, you will need to obtain permission directly from the copyright holder. To view a copy of this licence, visit http:// creativecommonshorg/licenses/by/4.0/.

\section{References}

Aki K, Richards PG (2002) Quantitative seismology. University Science, Sausalito

Aso N, Ohta K, Ide S (2016) Mathematical review on source-type diagrams. Earth Planets Space 68:52. https://doi.org/10.1186/s40623-016-0421-5

Boyd OS, Dreger DS, Lai VH, Gritto R (2015) A systematic analysis of seismic moment tensor at the Geysers geothermal field, California. Bull Seism Soc Am 105(6): 2969-2986. https://doi.org/10.1785/0120140285

Chapman CH (2019) Yet another moment-tensor parameterization. Geophysic Prospect 67:485-495. https://doi.org/10. $1111 / 1365-2478.12755$

Chapman CH, Leaney WS (2012) A new moment-tensor decomposition for seismic events in anisotropic media. Geophys J Int 188:343-370. https://doi.org/10.1111/j.1365246X.2011.05265.x

Cormen TH, Leiserson CE, Rivest RL, Stein C (2009) Introduction to algorithms. MIT Press, Cambridge

Eaton DW, Forouhideh F (2010) Microseismic moment tensors: the good, the bad and the ugly. GSEG Recorder 35(9):44-47

Hudson JA, Pearce RG, Rogers RM (1989) Source type plot for inversion of the moment tensor. J Geophys Res 94: 765-774. https://doi.org/10.1029/JB094iB01p00765
Jost ML, Herrmann RB (1989) A student's guide to and review of moment tensors. Seism Res Lett 60(2):37-57. https://doi.org/10.1785/gssrl.60.2.37

Julian B, Miller AD, Foulger GR (1998) Non-doublecouple earthquakes 1. Theor Rev Geophys 36(4):525-549. https://doi.org/10.1029/98RG00716

Kagan YY (2003) Accuracy of modern global earthquake catalogs. Phys Earth Planet Inter 135:173-209. https://doi.org/10.1016/S0031-9201(02)00214-5

Knopoff L, Randall MJ (1970) The compensated linear-vector dipole: a possible mechanism for deep earthquakes. J Geophys Res 75(26):4957-4963. https://doi.org/10.1029/ JB075i026p04957

Miller AD, Foulger GR, Julian B (1998) Non-double-couple earthquakes 2. Observations. Rev Geophys 36(4):551-568. https://doi.org/10.1029/98RG00717

Riedesel MA, Jordan TH (1989) Display and assessment of seismic moment tensors. Bull Seism Soc Am 79:85-100

Silver PG, Jordan TH (1982) Optimal estimation of the scalar seismic moment. Geophys J Roy Astr Soc 70:755-787. https://doi.org/10.1111/j.1365-246X.1982. tb05982.x

Tape W, Tape C (2012a) A geometric setting for moment tensors. Geophys J Int 190:476-498. https://doi.org/10.1111/j. 1365-246X.2012.05491.x

Tape W, Tape C (2012b) A geometric comparison of sourcetype plots for moment tensors. Geophys J Int 190:499-510. https://doi.org/10.1111/j.1365-246X.2012.05490.x

Tape W, Tape C (2015) A uniform parameterization of moment tensors. Geophys J Int 202:2074-2081. https://doi.org/10.1093/gji/ggv262

Tape W, Tape C (2019) The eigenvalue lune as a window on moment tensors. Geophys J Int 216:19-22. https://doi.org/10.1093/gji/ggy373

Vavryčuk V (2001) Inversion for parameters of tensile earthquakes. J Geophys Res 106(B8):16.339-16.355. https://doi.org/10.1029/2001JB000372

Vavryčuk V (2005) Focal mechanisms in anisotropic media. Geophys J Int 161:334-346. https://doi.org/10.1111/j.1365246X.2005.02585.x

Vavryčuk V (2015) Moment tensor decompositions revisited. J Seismol 19:231-252. https://doi.org/10.1007/s10950-0149463-y

Vavryčuk V, Hrubcová P (2017) Seismological evidence of fault weakening due to erosion by fluid from observations of intraplate. J Geophys Res Solid Earth 122:3701-3718. https://doi.org/10.1002/2017JB013958

Yu C, Vavryčuk V, Adamová P, Bohnhoff M (2018) Moment tensors of induced microearthquakes in the geysers geothermal reservoir from broadband seismic recordings: implications for faulting regime, stress tensor, and fluid pressure. J Geophys Res Solid Earth 123:8748-8766. https://doi.org/10.1029/2018JB016251

Zhu L, Ben-Zion Y (2013) Parametrization of general seismic potency and moment tensors for source inversion of seismic waveform data. Geophys J Int 194:839-843. https://doi.org/10.1093/gji/ggt137

Publisher's note Springer Nature remains neutral with regard to jurisdictional claims in published maps and institutional affiliations. 